\title{
Chest MRI to diagnose early diaphragmatic weakness in Pompe disease
}

\author{
Laurike Harlaar ${ }^{1}$, Pierluigi Ciet ${ }^{2}$, Gijs van Tulder ${ }^{3}$, Alice Pittaro ${ }^{4}$, Harmke A. van Kooten ${ }^{1}$,
}

Nadine A. M. E. van der Beek', Esther Brusse', Piotr A. Wielopolski ${ }^{4}$, Marleen de Bruijne ${ }^{3,5}$, Ans T. van der Ploeg ${ }^{6}$,

Harm A. W. M. Tiddens ${ }^{2}$ and Pieter A. van Doorn ${ }^{1 *}$

\begin{abstract}
Background: In Pompe disease, an inherited metabolic muscle disorder, severe diaphragmatic weakness often occurs. Enzyme replacement treatment is relatively ineffective for respiratory function, possibly because of irreversible damage to the diaphragm early in the disease course. Mildly impaired diaphragmatic function may not be recognized by spirometry, which is commonly used to study respiratory function. In this cross-sectional study, we aimed to identify early signs of diaphragmatic weakness in Pompe patients using chest MRI.
\end{abstract}

Methods: Pompe patients covering the spectrum of disease severity, and sex and age matched healthy controls were prospectively included and studied using spirometry-controlled sagittal MR images of both mid-hemidiaphragms during forced inspiration. The motions of the diaphragm and thoracic wall were evaluated by measuring thoracic cranial-caudal and anterior-posterior distance ratios between inspiration and expiration. The diaphragm shape was evaluated by measuring the height of the diaphragm curvature. We used multiple linear regression analysis to compare different groups.

Results: We included 22 Pompe patients with decreased spirometry results (forced vital capacity in supine position $<80 \%$ predicted); 13 Pompe patients with normal spirometry results (forced vital capacity in supine position $\geq 80 \%$ predicted) and 18 healthy controls. The mean cranial-caudal ratio was only 1.32 in patients with decreased spirometry results, 1.60 in patients with normal spirometry results and 1.72 in healthy controls $(p<0.001)$. Anterior-posterior ratios showed no significant differences. The mean height ratios of the diaphragm curvature were 1.41 in patients with decreased spirometry results, 1.08 in patients with normal spirometry results and 0.82 in healthy controls $(p=0.001)$, indicating an increased curvature of the diaphragm during inspiration in Pompe patients.

Conclusions: Even in early-stage Pompe disease, when spirometry results are still within normal range, the motion of the diaphragm is already reduced and the shape is more curved during inspiration. MRI can be used to detect early signs of diaphragmatic weakness in patients with Pompe disease, which might help to select patients for early intervention to prevent possible irreversible damage to the diaphragm.

Keywords: Pompe disease, Neuromuscular disease, Lysosomal storage disease, MRI, Diaphragm, Respiratory function

*Correspondence: p.a.vandoorn@erasmusmc.nl

${ }^{1}$ Center for Lysosomal and Metabolic Diseases, Department

of Neurology, Erasmus MC, University Medical Center Rotterdam, Dr. Molewaterplein 40, 3015 GD Rotterdam, The Netherlands

Full list of author information is available at the end of the article

\section{Background}

Respiratory insufficiency due to muscle weakness is often observed in patients with muscle disease, and is a typical characteristic of patients with advanced Pompe disease, an autosomal recessive metabolic myopathy caused by a deficiency of acid alpha-glucosidase [1-3]. Respiratory dysfunction in Pompe patients is caused mainly by original author(s) and the source, provide a link to the Creative Commons licence, and indicate if changes were made. The images or other third party material in this article are included in the article's Creative Commons licence, unless indicated otherwise in a credit line to the material. If material is not included in the article's Creative Commons licence and your intended use is not permitted by statutory regulation or exceeds the permitted use, you will need to obtain permission directly from the copyright holder. To view a copy of this licence, visit http://creativecommons.org/licenses/by/4.0/. The Creative Commons Public Domain Dedication waiver (http://creativeco mmons.org/publicdomain/zero/1.0/) applies to the data made available in this article, unless otherwise stated in a credit line to the data. 
weakness of the diaphragm, and is demonstrated by a decreased forced vital capacity (FVC) particularly in supine position $[4,5]$. In adults with non-classic Pompe disease, treatment with enzyme replacement therapy (ERT) resulted in an improved walking distance and muscle strength and stabilizing of respiratory function [6-10]. However, the effect of ERT is much smaller on respiratory function than on skeletal muscle function [7, 9, 10]. The international guideline for starting ERT, indicates that ERT should start when patients have skeletal muscle weakness and/or respiratory muscle weakness defined as an forced vital capacity (FVC) of below $80 \%$ predicted [11]. However, it is possible that damage to the diaphragm had already become irreversible before ERT was started. Early recognition of diaphragmatic weakness is important to allow a timely start of treatment, which is even more important as new therapies are currently tested or are in a preclinical phase.

Routine pulmonary function tests, such as vital capacity, FVC and mean inspiratory and expiratory pressures (MIP and MEP) do not differentiate between the function of the diaphragm and the intercostal muscles $[5,12,13]$. Improved insight into the contribution of the different respiratory muscles to inspiration can be provided using spirometry-controlled MRI, evaluating the entire diaphragm and thoracic wall during respiratory movements [14].

Using MRI, the predominantly involvement of the diaphragm in Pompe was shown in patients with advanced muscular involvement [15-18]. However, these studies do not reveal when diaphragmatic weakness starts, and whether an impaired diaphragm function is initially compensated by other respiratory muscles, resulting in normal pulmonary function tests results.

The aim of this study was to identify early signs of diaphragmatic weakness. We therefore used advanced image-analysis techniques to evaluate the motion and shape of the diaphragm in detail. We included a large group of children and adults with Pompe disease covering the spectrum of disease severity, ranging from those with normal spirometry results to those who need nocturnal ventilation due to respiratory dysfunction.

\section{Methods}

\section{Study design and participants}

In this cross-sectional study, we conducted spirometercontrolled MRI scans in Pompe patients (age $\geq 8$ years) and healthy controls who had been matched for sex and age. Pompe patients who visited the Center for Lysosomal and Metabolic Diseases at Erasmus MC University Medical Center, the reference center for Pompe disease in the Netherlands, were consecutively invited to participate between January 2016 and February 2018. Inclusion criteria were a confirmed diagnosis of non-classic Pompe disease (based on two disease causing variants in the acid alpha-glucosidase gene and/or decreased enzyme activity in fibroblasts) and the ability to lie in a supine position for at least $30 \mathrm{~min}$ without mechanical ventilation. Exclusion criteria were comorbidities or devices that did not permit MRI investigations, and claustrophobia. The protocol was approved by the Medical Ethical Committee at our hospital (MEC-2007-103, amendment 7). All participants provided written informed consent.

\section{Pulmonary function tests}

Before MRI, pulmonary function tests were performed according to ATS/ERS standards [19]. FVC was measured with the patient in upright seated and supine positions. MIP and MEP were measured with the patient in the upright position. All respiration maneuvers were repeated three times and the best effort was used in further analysis. Results were expressed as a percentage of predicted normal values [20,21].

\section{MRI analysis}

All patients were examined on a 3 T GE Signa 750 MRI (General Electric Healthcare, Milwaukee, Winconsin, USA), using the whole-body coil for radio-frequency excitation and a 32-channel torso coil for signal reception. We developed a new MRI scanning protocol including end-expiration and end-inspiration breath-hold acquisitions, followed by dynamic acquisitions during forced expiration, forced inspiration, and the sniff maneuver (Table 1). To ensure that all breathing maneuvers were executed correctly and with maximum effort, all breathing maneuvers were performed before and during MRI using a MR compatible spirometer, with instructions given through headphone. The overall acquisition time per patient was $20-25$ min.

In this study, we used dynamic sagittal bi-dimensional (2D) images, starting at breath holding at end-expiration, followed by a forced inspiration maneuver, and ending at breath holding at end-inspiration. The sagittal levels were manually selected at the right and left mid-hemidiaphragm using the three-dimensional (3D) breath holding acquisition at end-inspiration.

\section{Image segmentation}

We selected the images during the inspiration maneuver, comprising \pm 15 of 60 images. Manual image segmentation was performed by two observers (LH and AP) using ITK-SNAP (Version 3.6.0 Apr 1, 2017, Copyright (c) 1998-2017, www.itksnap.org) [22]. This free, opensource software application enables to manually indicate the area of the lung on the selected images. Anterior and posterior points of the insertion of the diaphragm were 
Table 1 MRI protocol

\begin{tabular}{|c|c|c|c|c|c|c|}
\hline Breathing & $\begin{array}{c}\text { Breath hold } \\
\text { end-inspiration }\end{array}$ & $\begin{array}{c}\text { Dynamic } \\
\text { forced }\end{array}$ & Dynamic & forced inspira & ation & $\begin{array}{l}\text { Dynamic sniff } \\
\text { maneuver }\end{array}$ \\
\hline Sequence & 3D SPGR & $\begin{array}{l}\text { 3D SPGR } \\
\text { DISCO }\end{array}$ & 2D FSPGR & $\begin{array}{c}\text { 3D } \\
\text { MULIPHASE } \\
\text { SPGR }\end{array}$ & $\begin{array}{l}\text { 3D SPGR } \\
\text { TRICKS }\end{array}$ & 3D SPGR DISCO \\
\hline Orientation & Sagittal & Sagittal & $\begin{array}{l}\text { Sagittal at right } \\
\text { and left mid- } \\
\text { hemi-diaphragm }\end{array}$ & Sagittal & Sagittal & Sagittal \\
\hline $\begin{array}{l}\text { Repetition time } \\
\text { (ms) }\end{array}$ & 1.2 & 1.0 & 2.3 & 1.0 & 1.0 & 1.0 \\
\hline Echo time (ms) & 0.5 & 0.3 & 0.8 & 0.3 & 0.4 & 0.3 \\
\hline Field of view $(\mathrm{cm})$ & 36 & 48 & 36 & 48 & 48 & 48 \\
\hline $\begin{array}{l}\text { Percent phase } \\
\text { field of view } \\
\text { sampling }\end{array}$ & & $50 \%$ & $75 \%$ & $50 \%$ & $50 \%$ & $50 \%$ \\
\hline Matrix & $120 \times 120$ & $48 \times 48$ & $100 \times 100$ & $48 \times 48$ & $48 \times 48$ & $48 \times 48$ \\
\hline Flip angle & $2^{\circ}$ & $1^{\circ}$ & $7^{\circ}$ & $1^{\circ}$ & $1^{\circ}$ & $1^{\circ}$ \\
\hline $\begin{array}{c}\text { Voxel-resolution } \\
(\mathrm{mm})\end{array}$ & $3 \times 3 \times 3$ isotropic & $\begin{array}{c}10 \times 10 \times 10 \\
\text { isotropic }\end{array}$ & $3.6 \times 3.6 \times 10$ & $\begin{array}{c}10 \times 10 \times 10 \\
\text { isotropic }\end{array}$ & $\begin{array}{c}10 \times 10 \times 10 \\
\text { isotropic }\end{array}$ & $\begin{array}{c}10 \times 10 \times 10 \\
\text { isotropic }\end{array}$ \\
\hline $\begin{array}{c}\text { Temporal } \\
\text { resolution (ms) }\end{array}$ & & 250 & 200 & 785 & 260 & 250 \\
\hline Scan time (s) & $6-10$ & 10 & 11 & 12 & $2-14$ & 10 \\
\hline Number of slices & $\begin{array}{l}\text { Depending on } \\
\text { the size of } \\
\text { patient }\end{array}$ & 42 & 1 right and 1 left & $\begin{array}{l}\text { Depending on } \\
\text { the size of } \\
\text { patient }\end{array}$ & 42 & 42 \\
\hline
\end{tabular}

MRI protocol and parameters on 3 T GE Signa 750 MRI (General Electric Healthcare, Milwaukee, USA) with a 32-channel torso coil. The acquisition for this part of the study is marked with thick borders and included 2D dynamic inspiratory SPGR multiphase sagittal acquisition at the level of the right and left mid-hemi-diaphragm. All dynamic inspiration acquisitions started with breath holding at end-expiration and ended with breath holding at end-inspiration. Dynamic forced expiration started with breath holding at end-inspiration and ended with breath holding at end-expiration

$2 \mathrm{D}=$ bi-dimensional, $3 \mathrm{D}=$ three-dimensional, Disco = DIfferential Sub-sampling with Cartesian Ordering, SPGR $=$ spoiled gradient echo, Tricks $=$ time-resolved imaging of contrast kinetics, FSGPR = fast spoiled gradient echo

a After respective 3D SPGR inspiratory and expiratory localizer sequences

b Sequence will be repeated after 2D FSPGR sequence

chosen as reference points for basal lung contours. For the left lung, the heart was included in the segmented lung area to optimally delineate the contours of the diaphragm (Fig. 1a, b).

\section{Automatic measurements}

The segmentations were analyzed with a custom-built Python script (Python 3.6.3, https://www.python.org/, (C2001-2019. Python Software Foundation; SciPy 1.1.0, https://www.scipy.org/, @2003-2019 SciPy developers) (Fig. 1c). The algorithm first determines the general orientation of the lung, to correct for minor differences in position between patients, by fitting an ellipse to the points on the lung outline. The lung apex is then defined as the highest point on the lung outline, measured in the direction of the major axis of the ellipse. The algorithm then detects the anterior and posterior costophrenic angles of the diaphragm, defined as the points with the largest distance to the centroid (center of gravity) in the lower part of the lungs. The diaphragm apex was defined as the point on the diaphragm contour that is furthest away from the linear line between both costophrenic angles of the diaphragm. Because for some patients this maximum-distance point was quite variable between consecutive frames, we used a derived diaphragm apex: therefore, the relative position of the diaphragm apex along the diaphragm contour was computed in each frame as a percentage of the diaphragm length; then, the median position of all frames was computed and this median relative distance was used for each frame to compute the derived diaphragm apex. All reference points 
were visual inspected by LH and GT to confirm that the points were correctly indicated.

\section{MRI outcome measures}

Four thorax-related outcomes and four diaphragmrelated outcomes were measured at end-expiration and end-inspiration (Fig. 1d, e). To correct for anatomical variations between individual patients, we calculated ratios by dividing end-inspiration outcomes by end-expiration outcomes.

\section{Thorax-related outcomes}

The cranial-caudal (CC) distance was measured as the distance between the lung apex and diaphragm apex to evaluate diaphragmatic motion. The anterior-posterior (AP) distance is the longest distance from the front to the back of the lung to evaluate motion of the thoracic wall. To calculate the diaphragm motion relative to the motion of the thoracic wall, the CC-AP ratio was calculated by dividing the $\mathrm{CC}$ ratio by the $\mathrm{AP}$ ratio. The lung area is the area of the segmentation to evaluate the result of all respiratory muscles together. For the left hemi-diaphragm, lung area included the area of the heart to reduce the effect of heart size.

\section{Diaphragm-related outcomes}

To evaluate the shape of the diaphragm, the area below the diaphragm curvature (DA) and the height of the diaphragm curvature $(\mathrm{DH})$ were measured to calculate the DA ratio and DH ratio between inspiration and expiration. The DA is the area between the diaphragm contour and the linear line connecting both diaphragm corners. The $\mathrm{DH}$ is the perpendicular distance between the diaphragm apex and the linear line. In addition, the absolute displacements of the anterior and posterior costophrenic angles of the diaphragm were measured in consecutive images from end-expiration to end-inspiration.

\section{Validation of measurements}

To validate automatic measurements, manual measurements of thorax related outcomes were performed by two independent observers ( $\mathrm{LH}$ and $\mathrm{AP}$ ). CC distance and AP distance were measured using ITK-SNAP and lung area was measured with Aw Server (2.0 General Electric Healthcare ( $)$ 2009-2012). All automatically measured thorax-related outcomes were compared to manually measured outcomes. Second, both observers performed all measurements twice in 5 patients (9\%) to calculate intra-observer variability and in 10 similar patients (19\%) to calculate inter-observer variability. These measurements included new segmentations on end-expiration and end-inspiration images. We used intra-class correlation coefficients (ICC) based on absolute-agreement and two-way mixed-effects model [23]. Bland-Altman plots were inspected to assess possible systematic errors.

\section{Statistical analysis}

To investigate early signs of diaphragmatic weakness, we subdivided the Pompe patients into two subgroups: patients with normal spirometry results (FVC supine $\geq 80 \%$ than predicted and $\mathrm{z}$-score $\geq-1.64$ ) and patients with decreased spirometry results (FVC supine $<80 \%$ and $\mathrm{z}$-score $<-1.64$ ).

To analyze differences in patient characteristics and pulmonary function outcomes between the two subgroups of Pompe patients and the healthy controls, we used the chi-square test for categorical variables and the Kruskal Wallis test for continuous variables. The MannWhitney test was used to test for differences in disease duration and duration of ERT between the two subgroups of Pompe patients. Overall differences in MRI outcomes and differences between healthy controls and the two subgroups of Pompe patients were analyzed using multiple linear regression analysis, with adjustment for significant differences in patient characteristics between the three subgroups. Residual plots were inspected to check the linearity assumption. The Spearman correlation coefficient was used to calculate the strength of association between the pulmonary function test outcomes and the MRI outcomes.

All analyses were performed on the left and right sides of the thorax. Because segmentation of the left hemidiaphragm is more cumbersome due to the heart, we report only the results of the right hemidiaphragm.

Statistical analysis was performed with SPSS for Windows (version 25, SPSS Inc, Chicago, IL). The significance level was set at $p \leq 0.05$. Unless indicated otherwise, we report overall differences between the three subgroups. Differences between subgroups were corrected for multiple testing using Bonferroni's method, resulting in a significance level of $p \leq 0.017$. Due to the explorative

\footnotetext{
(See figure on next page.)

Fig. $1 \mathrm{MRI}$ measurements and outcomes. a, b Examples of manual image segmentation in the right and left lung at expiration and inspiration in an healthy control. c Steps of the automatic algorithm to define reference points to calculate the different outcome measures. $\mathbf{d}$ and $\mathbf{e}$ Thorax- and diaphragm-related outcomes. The grey lines depict the outlines of the lung in consecutive images from end expiration to end-inspiration. The red point indicates the anterior corner of the diaphragm and the blue point the posterior corner of the diaphragm. The green points indicate displacement of the lung apex and diaphragm apex. Purple arrows and purple areas indicate the outcome measurements
} 


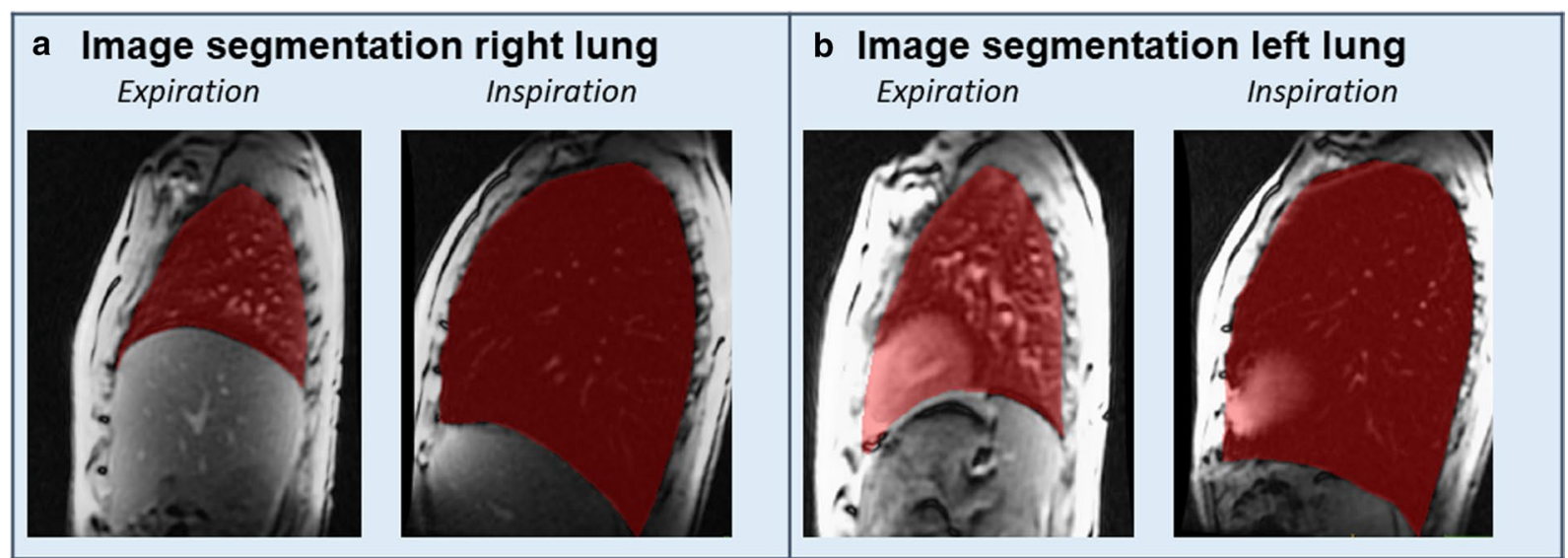

\section{c Automatic measurements}

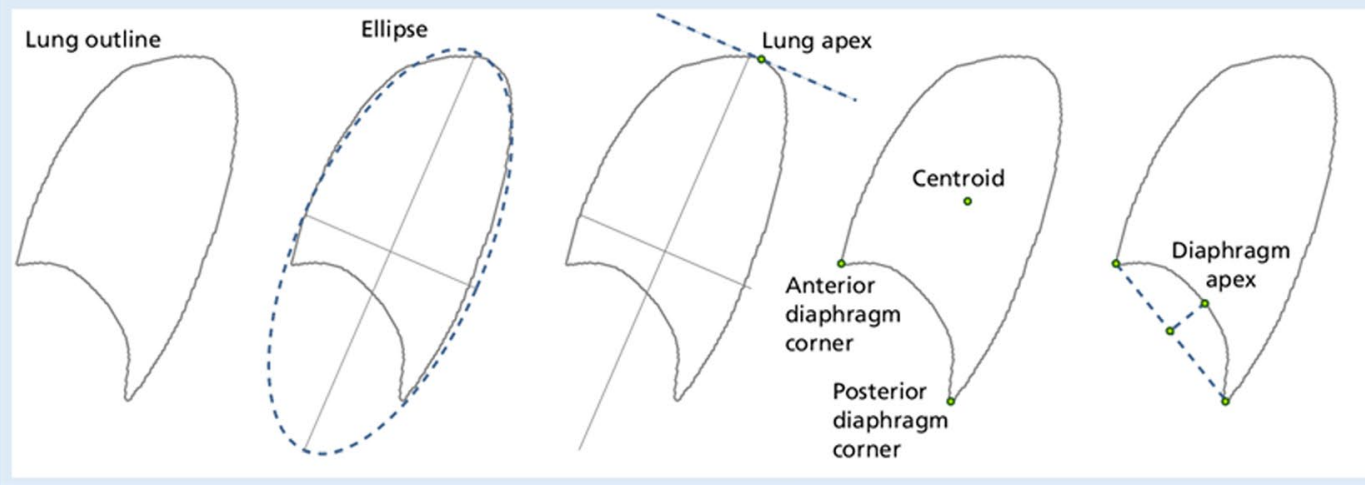

\section{Cranial-caudal ratio (CC ratio) \\ Anterior-posterior ratio (AP ratio)}

d Thorax-related outcomes

Lung area ratio

$\mathrm{CC}$ ratio / $\mathrm{AP}$ ratio (CC-AP ratio)

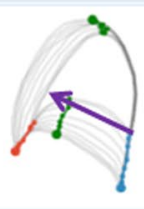

Expiration

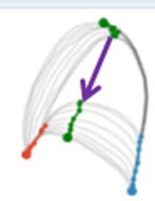

Inspiration
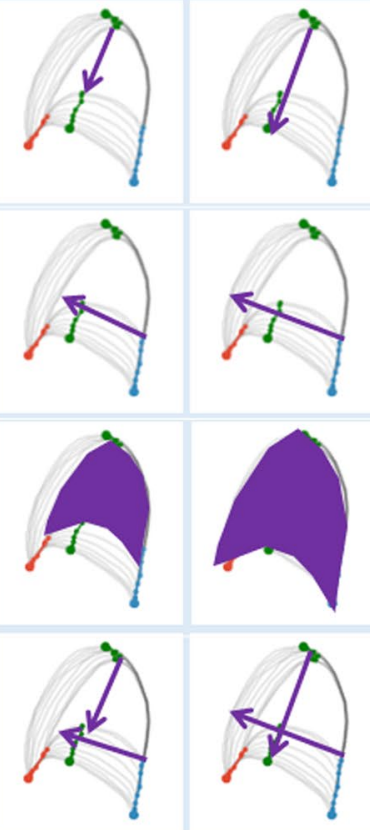

e Diaphragm-related outcomes

Expiration Inspiration

Area under the diaphragm curvature ratio

(DA ratio)
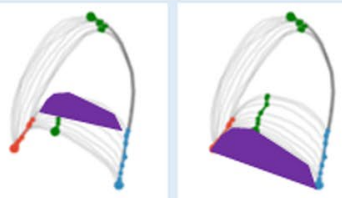

Height of the

diaphragm curvature ratio

(DH ratio)

Absolute displacement of the anterior corner of the diaphragm (anterior displacement)
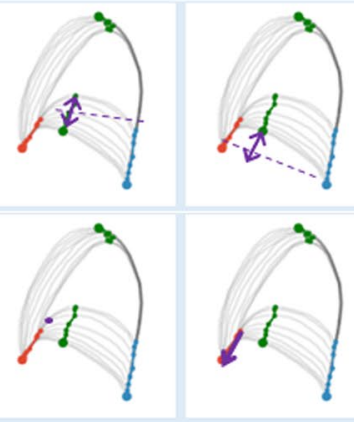

Absolute displacement of the posterior corner of the diaphragm (posterior displacement) 
Table 2 Participant characteristics

\begin{tabular}{|c|c|c|c|c|}
\hline & $\begin{array}{l}\text { Pompe patients with decreased } \\
\text { spirometry results } \\
\text { (FVC supine } \\
<80 \%)(n=22)\end{array}$ & $\begin{array}{l}\text { Pompe patients with normal } \\
\text { spirometry results } \\
\text { (FVC supine } \\
\geq 80 \%)(n=13)\end{array}$ & $\begin{array}{l}\text { Healthy controls } \\
(n=18)\end{array}$ & $p$ value \\
\hline Sex, number of males (\%) & $11(50 \%)$ & $7(54 \%)$ & $8(44 \%)$ & 0.869 \\
\hline Age, years & $45 \pm 16$ & $31 \pm 14$ & $43 \pm 14$ & 0.030 \\
\hline Height, cm & $178 \pm 11$ & $174 \pm 9$ & $178 \pm 12$ & 0.761 \\
\hline Weight, kg & $76 \pm 16$ & $70 \pm 13$ & $79 \pm 14$ & 0.291 \\
\hline $\mathrm{BMI}, \mathrm{kg} / \mathrm{m}^{2}$ & $24 \pm 4$ & $23 \pm 4$ & $25 \pm 3$ & 0.262 \\
\hline Disease duration, years & $16 \pm 8$ & $9 \pm 9$ & - & 0.018 \\
\hline Patients on ERT, n (\%) & $18(82 \%)$ & $6(46 \%)$ & - & 0.028 \\
\hline Duration of ERT, years & $6 \pm 5$ & $6 \pm 4$ & - & 0.811 \\
\hline Non-invasive ventilation, n (\%) & $1(3 \%)$ & $0(0 \%)$ & - & 0.435 \\
\hline FVC upright, $\%$ of predicted & $81 \pm 11$ & $97 \pm 9$ & $106 \pm 8$ & $<0.001$ \\
\hline FVC supine, $\%$ of predicted & $60 \pm 12$ & $94 \pm 9$ & $102 \pm 8$ & $<0.001$ \\
\hline$\triangle F V C, \%$ of predicted & $21 \pm 10$ & $4 \pm 4$ & $4 \pm 4$ & $<0.001$ \\
\hline MIP, \% of predicted & $72 \pm 23$ & $88 \pm 28$ & $106 \pm 25$ & 0.001 \\
\hline MEP, $\%$ of predicted & $80 \pm 28$ & $81 \pm 28$ & $112 \pm 28$ & 0.002 \\
\hline
\end{tabular}

Characteristics and outcomes of pulmonary function tests of Pompe patients and healthy controls. Continues values are presented as mean \pm standard deviation and were tested using the Mann Whitney or Kruskal Wallis tests. Categorical values are presented as number with percentage and were tested with the chi square test. P-values indicate overall differences between groups

$\mathrm{BMI}=$ body mass index, $\mathrm{ERT}=$ enzyme replacement therapy, $\mathrm{FVC}=$ forced vital capacity, $\triangle \mathrm{FVC}=\mathrm{FVC}$ upright $-\mathrm{FVC}$ supine, MIP $=$ maximum inspiratory pressure, $\mathrm{MEP}=$ maximum expiratory pressure

character of the study, we did not adjust for multiple testing when reporting the different MRI outcomes.

\section{Results}

\section{Participants characteristics and pulmonary function tests}

We included 35 patients with Pompe disease (age 15-70 years) and 18 sex and age-matched healthy controls (Table 2). Thirteen patients had spirometry results in the normal range (FVC supine $\geq 80 \%$ ); none of these patients had significant dyspnea or signs of nocturnal hypoventilation. Twenty-two patients had decreased spirometry results (FVC supine $<80 \%$ ), one of whom used nocturnal non-invasive ventilation due to hypercapnia. Patients with normal spirometry results were younger, had a shorter disease duration since symptom onset, and fewer of them had been treated with ERT than patients with decreased spirometry results. Healthy controls had better FVC, MIP and MEP outcomes than Pompe patients. None of the patients had a significant kyphoscoliosis needing surgery or referral to an orthopedic surgeon.

\section{Thorax-related outcomes}

The mean CC ratios were as follows: 1.72 in healthy controls, 1.60 in Pompe patients with normal spirometry results, and 1.32 in Pompe patients with decreased spirometry results $(p<0.001)$. The mean AP ratio did not differ significantly between the three subgroups. The mean lung area ratios were 2.54 in healthy controls, 2.57 in Pompe patients with normal spirometry results, and 1.95 in Pompe patients with decreased spirometry results $(p=0.001)$. The mean CC-AP ratios were 1.38 in healthy controls, 1.22 in Pompe patients with normal spirometry results, and 1.04 in Pompe patients with decreased spirometry results $(p<0.001)$. While patients with normal spirometry results had lower $\mathrm{CC}$ ratios and CC-AP ratios than healthy controls $(p=0.002$ and $p=0.005)$, their lung area ratios were not significantly different (Fig. 2).

The agreement between automatic and manual measurements was excellent (ICC range 0.93-0.99), and the intra- and inter-observer agreement between manual measurements was good to excellent (ICC range $0.80-0.99)$.

\section{Diaphragm-related outcomes}

During inspiration, DA and DH in healthy controls decreased, indicating a decreased curvature of the diaphragm. In Pompe patients, however, the curvature of the diaphragm remained equal or increased during inspiration. In healthy controls mean DA ratio was 0.91 and mean DH ratio 0.82, in Pompe patients with normal spirometry results mean DA ratio was 1.34 and mean $\mathrm{DH}$ ratio was 1.08 , and in those with decreased spirometry results was mean DA ratio was $1.79(p<0.01)$ and the mean DH ratio was $1.41(p<0.01)$. In healthy controls, the absolute displacement of the posterior costophrenic 

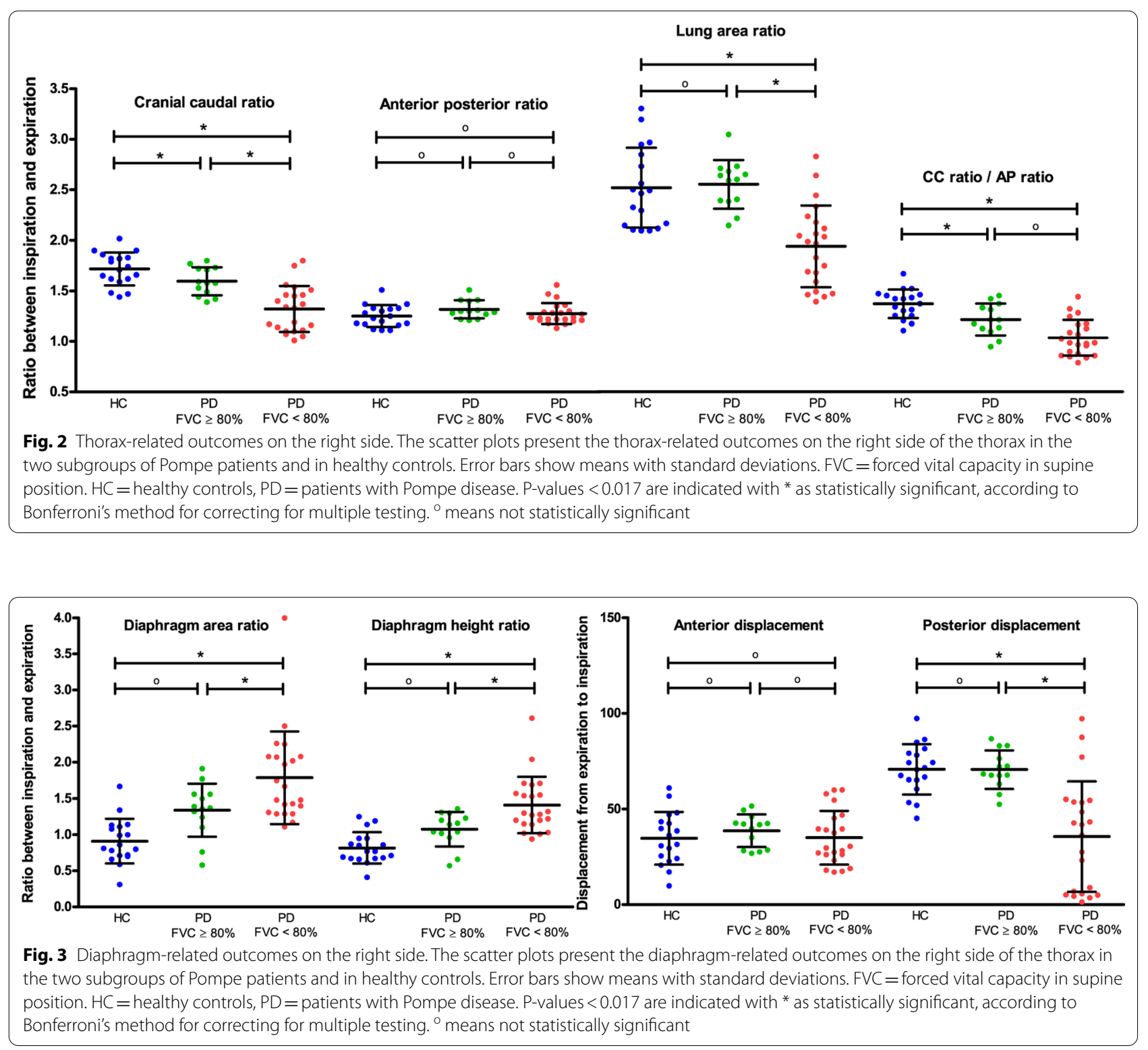

angle of the diaphragm was larger than the displacement of the anterior costophrenic angle. Mean absolute displacement of the posterior costophrenic angle was $70.7 \mathrm{~mm}$ in healthy controls, $70.6 \mathrm{~mm}$ in Pompe patients with normal spirometry results, and $35.6 \mathrm{~mm}$ in Pompe patients with decreased spirometry results $(p<0.001)$. Mean absolute displacement of the anterior costophrenic angle did not differ between healthy controls and the two groups of Pompe patients. In patients with normal spirometry results, the DA and $\mathrm{DH}$ ratios were higher than in healthy controls $(p=0.088$ and $p=0.034$, which was not significant after Bonferroni's correction) (Fig. 3). 


\section{Levels of diaphragmatic weakness}

Using MRI to assess diaphragmatic weakness, different levels of severity could be indicted (Fig. 4). In patients with severe diaphragmatic weakness, the diaphragm shows hardly any motion and the $\mathrm{CC}$ ratio is $\sim 1$. Those with moderate diaphragmatic weakness still have diaphragmatic motion (CC ratio $>1$ ), although it is lower than that in healthy controls. In patients with mild diaphragmatic weakness, the CC ratio is normal, but DA ratio and $\mathrm{DH}$ ratio are increased, indicating an increased curvature of the diaphragm compared to in healthy controls and patients without diaphragmatic weakness. Importantly, in patients with mild or moderate diaphragmatic weakness, AP ratio is still normal or increased, indicating an increased motion of the thoracic wall. The large contribution to inspiration of the thoracic wall relative to that of the diaphragm is also reflected by a lower CC-AP ratio.

\section{Correlation between pulmonary function tests and MRI-outcomes}

$\mathrm{CC}$ ratio, $\mathrm{CC}$ - $\mathrm{AP}$ ratio and absolute displacement of the posterior corner of the diaphragm, outcomes evaluating the motion of the diaphragm, showed the best correlations with FVC supine $(>0.64)$ and $\triangle \mathrm{FVC}(<-0.59)$ (Table 3). The correlations of these MRI outcomes with FVC upright and MIP, which lay between 0.32 and 0.52 , showed that upright pulmonary function tests provided less information on the motion of the diaphragm than supine pulmonary function tests. The MRI outcomes that measure the curvature of the diaphragm during inspiration-the DA and DH ratios-showed moderate correlations with FVC supine $(<-0.58)$ and $\triangle \mathrm{FVC}(>0.48)$. The low correlations of MEP with all MRI outcomes $(<0.3)$ indicate that this test does not have the sensitivity necessary for measuring diaphragmatic motion or shape.

\section{Discussion}

In this cross-sectional study we used 2D sagittal dynamic MRI to evaluate the motion and shape of the diaphragm in children and adults with non-classic Pompe disease who had normal or decreased spirometry results. We found that motion of the diaphragm is decreased in patients with Pompe disease, and that the curvature of the diaphragm in these patients is increased during inspiration. Interestingly, these MRI signs were observed even in Pompe patients whose spirometry results were still within the normal range. This means that MRI enables to detect early signs of diaphragmatic weakness. These results are important for Pompe patients, but potentially also for patients with other neuromuscular disease having respiratory dysfunction, because early recognition of impaired diaphragmatic function enables to start early interventions to prevent irreversible damage to the diaphragm.

Our observations show a clear dissociation between the loss of function of the diaphragm and the intercostal musculature. Diaphragmatic weakness is common in adults with Pompe disease, and earlier 2D and 3D MRI pilot studies in small groups of Pompe patients with decreased spirometry results also found decreased motion of the diaphragm [15-18]. Interestingly, this is different compared to studies in patients with Duchenne muscular dystrophy, in whom the motion of the chest wall as well as the diaphragm are reduced [24]. As we wished to investigate whether early signs of diaphragmatic weakness could also be observed in Pompe patients with normal spirometry results, we conducted the current MRI study. A remarkable finding was that while motion of the thoracic wall still lay in the normal range, motion of the diaphragm was already less than in healthy controls. Early involvement of the diaphragm might be an explanation for the presentation with respiratory symptoms without limb girdle muscle weakness in in a subset of patients with Pompe disease [25, 26].

A second finding was that Pompe patients had an increased curvature of the diaphragm during inspiration compared to healthy controls. We hypothesize that the aberrant shape of the diaphragm during inspiration is caused by diaphragmatic weakness. In healthy controls, during inspiration, intrathoracic pressure is reduced and abdominal pressure is increased by the downward movement of the diaphragm and the outward movement of the chest wall $[27,28]$. In patients with severe diaphragmatic weakness, however, the lower intrathoracic pressure and impaired tonus of the diaphragm result in an upward paradoxical displacement of the diaphragm [28]. As many Pompe patients also have abdominal muscle weakness,

\footnotetext{
(See figure on next page.)

Fig. 4 Levels of diaphragmatic weakness. Examples of a healthy control and of four Pompe patients with different levels of diaphragmatic weakness. The left-hand images show the sagittal MRI at end-expiration and end-inspiration. The grey outlines show the contours of the lungs from expiration to inspiration (bold lines). The blue point is the posterior corner of the diaphragm and the red point the anterior corner. The green points show the lung apex and diaphragm apex. The individual results of pulmonary function tests (\% of predicted outcome) and MRI outcome ratios are also reported, with red numbers indicating abnormal outcomes compared to healthy controls. FVC $=$ forced vital capacity, $\triangle \mathrm{FVC}=\mathrm{FVC}$ upright FVC supine, MIP = maximum inspiration pressure, MEP = maximum expiration pressure, $C C$ ratio = cranial-caudal ratio, $\mathrm{AP}$ ratio $=$ anterior posterior ratio, $C$ - $A P$ ratio $=C C$ ratio $/ \mathrm{AP}$ ratio, $\mathrm{DH}$ ratio $=$ diaphragm height ratio, $\mathrm{DA}$ ratio $=$ diaphragm area ratio
} 


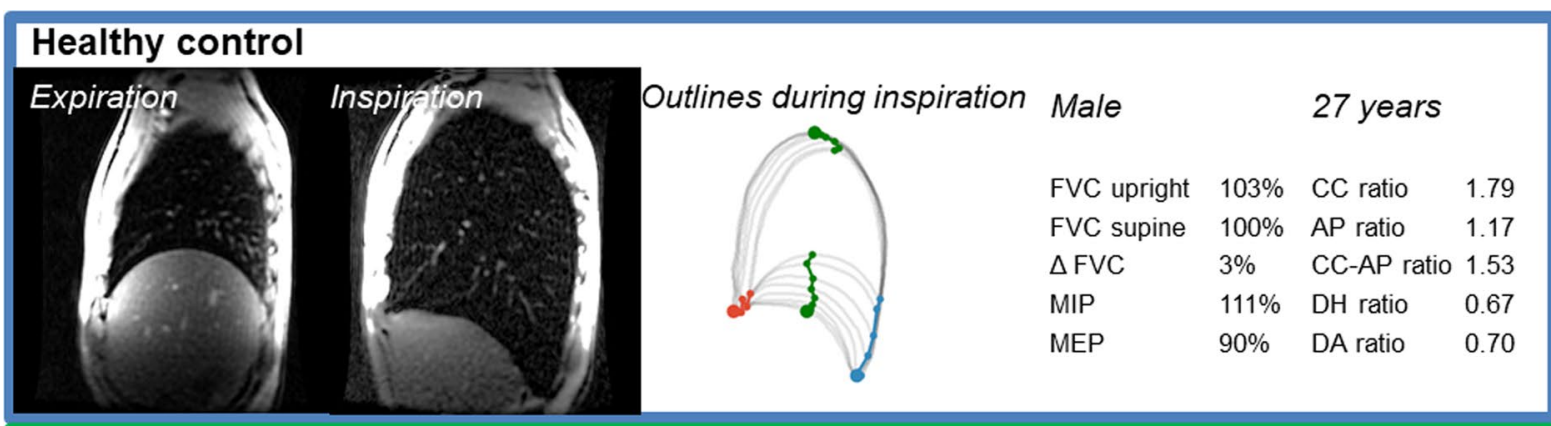

\section{Pompe patient - no diaphragmatic weakness}

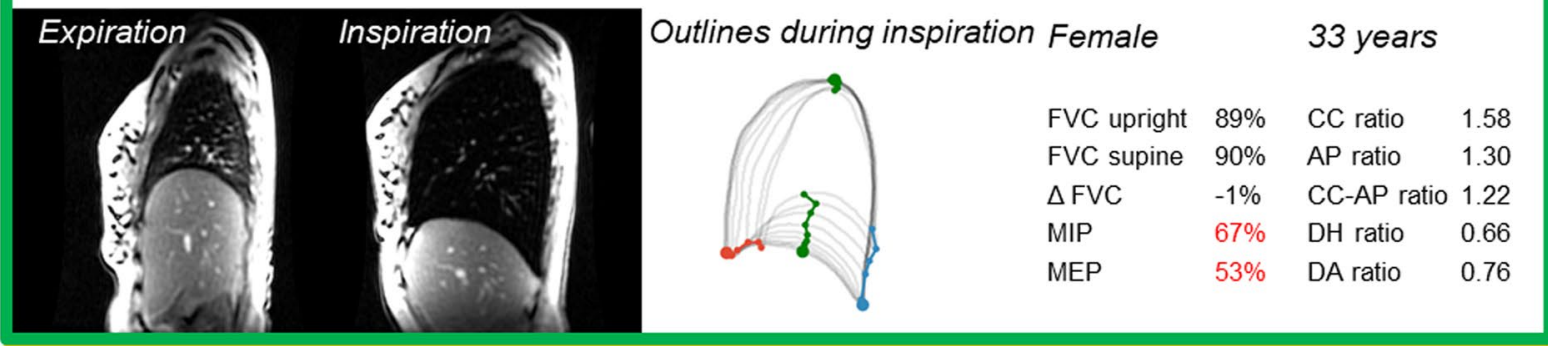

\section{Pompe patient - mild diaphragmatic weakness}

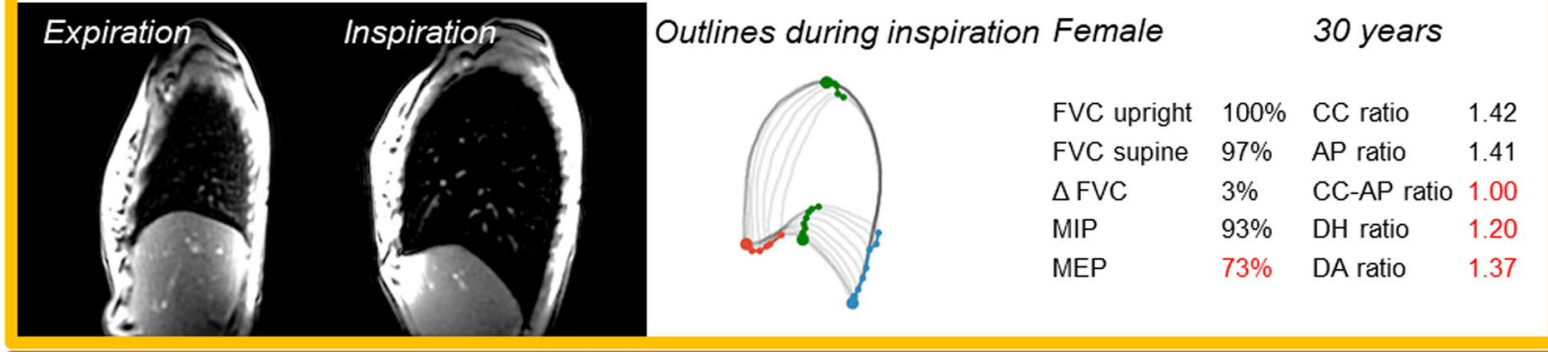

\section{Pompe patient - moderate diaphragmatic weakness}

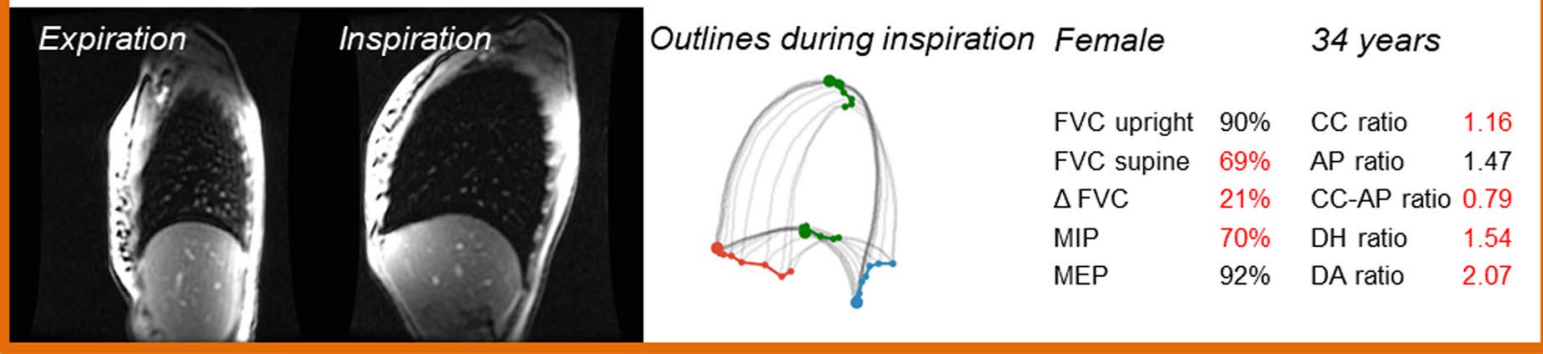

\section{Pompe patient - severe diaphragmatic weakness}

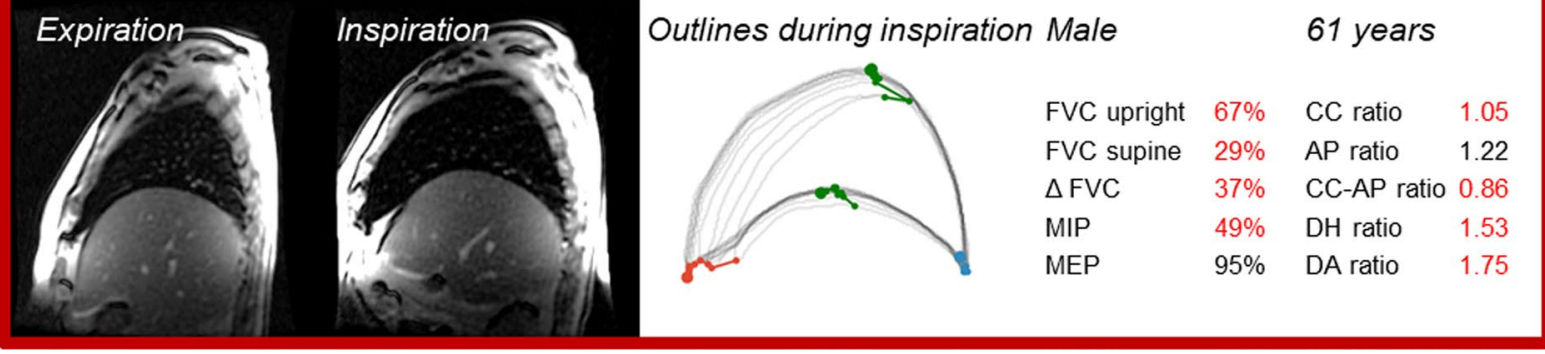


Table 3 Correlation between MRI outcomes and pulmonary function tests outcomes

\begin{tabular}{|c|c|c|c|c|c|}
\hline & FVC upright & FVC supine & $\Delta \mathrm{FVC}$ & MIP & MEP \\
\hline \multicolumn{6}{|l|}{ Thorax-related outcomes } \\
\hline Cranial-caudal ratio & $0.518^{* * *}$ & $0.680^{* * *}$ & $-0.654^{* * *}$ & $0.435^{* *}$ & 0.187 \\
\hline Anterior-posterior ratio & 0.035 & 0.097 & -0.112 & -0.008 & 0.040 \\
\hline Lung area ratio & $0.473^{* * *}$ & $0.636^{* * *}$ & $-0.610^{* * *}$ & $0.398^{* *}$ & 0.151 \\
\hline CC-AP ratio & $0.509^{* * *}$ & $0.643^{* * *}$ & $-0.608^{* * *}$ & $0.403^{* *}$ & 0.161 \\
\hline \multicolumn{6}{|c|}{ Diaphragm-related outcomes } \\
\hline Diaphragm area ratio & $-0.556^{* * *}$ & $-0.588^{* * *}$ & $0.484^{* * *}$ & $-0.355^{* *}$ & $-0.293^{*}$ \\
\hline Diaphragm height ratio & $-0.538^{* * *}$ & $-0.608^{* * *}$ & $0.552^{* * *}$ & $-0.382^{* *}$ & -0.270 \\
\hline Anterior displacement & 0.089 & 0.201 & -0.234 & -0.065 & -0.042 \\
\hline Posterior displacement & $0.486^{* * *}$ & $0.650^{* * *}$ & $-0.598^{* * *}$ & $0.322^{*}$ & 0.021 \\
\hline
\end{tabular}

Spearman correlation coefficients between thorax-related outcomes and diaphragm-related outcomes (ratios between end-inspiration and end-expiration outcomes) and outcomes of pulmonary function tests (\% predicted). Significant correlations are indicated with ${ }^{*} p<0.05,{ }^{* *} p<0.01$ or ${ }^{* * *} p<0.001$

$\mathrm{CC}-\mathrm{AP}$ ratio $=\mathrm{cranial}$ caudal ratio $/$ anterior posterior ratio. $\mathrm{FVC}=$ forced vital capacity, $\triangle \mathrm{FVC}=\mathrm{FVC}$ upright $-\mathrm{FVC}$ supine, $\mathrm{MEP}=\mathrm{mean}$ expiratory pressure, $\mathrm{MIP}=\mathrm{mean}$ inspiratory pressure

abdominal pressure will not increase sufficiently during inspiration. Consequently, there is little or no paradoxical movement of the diaphragm, but only a paradoxical increased curvature of the diaphragm during inspiration.

An important question is why the preferential weakness of the diaphragm compared to the intercostal muscles occurs. In healthy adults, the diaphragm comprises an equal distribution of slow-twitch type-1 fibers and fast-twitch type-2 fibers, while the intercostal muscles contain a higher proportion of fast-twitch type-2 fibers $[27,29,30]$. In Pompe patients, it is known that damage to skeletal muscle includes both muscle fiber types, but there have been no studies on the involvement of specific respiratory-muscle fiber types [31]. Early damage of the diaphragm however could also be facilitated by the fact that this muscle and tendon plate is relatively thin [32].

As our study showed that FVC supine and the difference between FVC upright and FVC supine $(\triangle \mathrm{FVC})$ had the highest correlations with the motion of the diaphragm as measured with MRI, these pulmonary function tests are important parameters to diagnose diaphragmatic weakness. While weakness of the diaphragm is usually clear in patients with FVC supine $<80 \%$, our dynamic MRI protocol can be used to demonstrate a different shape or reduced motion of the diaphragm in patients with an $\mathrm{FVC} \geq 80 \%$, which is considered to be in the normal range [11].

Ultrasound is another technique to investigate the diaphragm. In Pompe patients, it showed a reduced diaphragmatic thickness and motion and a good correlation with FVC in seated and supine position [33]. While ultrasound logistically might be more easy to perform, a comparison between diaphragmatic motion and motion of intercostal muscles and a more detailed evaluation of the curvature of the diaphragm is not possible. Therefore, detection of slight diaphragmatic weakness in an early stage of Pompe disease using ultrasound will likely be difficult.

By using our new 2D sagittal dynamic MRI protocol, we were able to identify reliable automatically measured outcome measures to characterize the motion and shape of the diaphragm. Our automatic measurements are operator independent and showed excellent correlation with manual measurements.

Our study however has some limitations. Spirometry-controlled MRI is logistically more difficult and expensive than regular pulmonary function tests. Second, segmentations were performed manually and were time consuming. Further development of automatic measurements, including automatic segmentations would be useful. Additional investigation is required to determine whether this chest MRI technique may also be useful to detect a potential neuromuscular cause in patients who present with dyspnea and/or respiratory failure of unknown origin. To generate reference values for our MRI outcomes, the population of healthy controls should be further increased.

\section{Conclusions}

We have demonstrated that MRI is a sensitive tool for detecting early stages of diaphragmatic weakness in patients with Pompe disease, even when spirometry results are within the normal range. MRI provides insight into the process of increasing diaphragmatic weakness, and when FVC starts to decline, possible irreversible functional changes of the diaphragm may have already taken place. This may explain 
why respiratory function as assessed by spirometry responds relatively poorly to ERT. Potentially, the MRI outcome measures may be helpful to optimize the start of treatment, and to assess diaphragmatic function and therapy response over time, not only in Pompe disease, but also in other neuromuscular diseases associated with respiratory dysfunction.

\begin{abstract}
Abbreviations
2D: Bi-dimensional; 3D: Three-dimensional; AP: Anterior-posterior; CC: Cranial-caudal; DA: Diaphragm area; DH: Diaphragm height; ERT: Enzyme Replacement Therapy; FVC: Forced Vital Capacity; ICC: Intra-class Correlation Coefficients; MEP: Maximum Expiratory Pressure; MIP: Maximum Inspiratory Pressure.
\end{abstract}

\section{Acknowledgements}

We thank prof. Dimitris Rizopoulos (Erasmus MC, University Medical Center Rotterdam, Department of Biostatistics) for help with the statistical analyses of the results and David Alexander for critically reviewing the manuscript.

\section{Authors' contributions}

LH, PC, HAK, NAMEB, EB, MB, ATP, HAWMT and PAD were involved in the design of the study. $L H, P C$ and PAW developed the MRI scanning protocol under supervision of MB and HAWMT. LH, HAK, NAMEB, EB, ATP and PAD recruited participants for the study. $L H, P C, G T$, AP performed MRI and data analysis under overall supervision of MB, ATP, HAWMT and PAD who also wrote the grant. All authors were involved in interpretation of the outcomes and drafting or revising the manuscript. All authors read and approved the final manuscript.

\section{Funding}

This work is supported by a grant from the Prinses Beatrix Spierfonds for neuromuscular disease (grant number W.OR15-10).

\section{Availability of data and materials}

The datasets containing anonymized MRI outcomes during and/or analysed during the current study, anonymized image segmentations and the algorithm to analyze segmentations are available from the corresponding author on reasonable request. In order to protect privacy and confidentiality of the study participants, complete MRI data is not publicly available.

\section{Ethics approval and consent to participate}

The protocol was approved by the Medical Ethical Committee at our hospital (MEC-2007-103, amendment 7). All participants provided written informed consent

\section{Consent for publication}

Not applicable.

\section{Competing interests}

NAMEB received funding for research and as advisor or speaker from SanofiGenzyme, under agreements with Erasmus MC University Medical Center. ATP received funding for research and/or clinical trials and as advisor or speaker from Amicus, Sanofi-Genzyme, Ultragenix, Spark Therapeutics, Audentes, Biomarin, Shire, Ciesi, Sarepta under agreements with Erasmus MC University Medical Center and the relevant industry. HAWMT received unconditional research grants outside the submitted work from Roche, Novartis, CFF, Vertex, Chiesi, Vectura, and Gilead and participated in the last 5 years in expert panels for Vertex and Gilead. All financial aspects for the grants are handled by Sophia Research BV. All other authors report no relevant disclosures.

\section{Author details}

${ }^{1}$ Center for Lysosomal and Metabolic Diseases, Department of Neurology, Erasmus MC, University Medical Center Rotterdam, Dr. Molewaterplein 40, 3015 GD Rotterdam, The Netherlands. ${ }^{2}$ Departments of Radiology and Nuclear Medicine, Paediatrics, and Respiratory Medicine and Allergology, Erasmus MC, University Medical Center Rotterdam, Rotterdam, The
Netherlands. ${ }^{3}$ Biomedical Imaging Group Rotterdam, Department of Radiology and Nuclear Medicine, Erasmus MC, University Medical Center Rotterdam, Rotterdam, The Netherlands. ${ }^{4}$ Department of Radiology and Nuclear Medicine, Erasmus MC, University Medical Center Rotterdam, Rotterdam, The Netherlands. ${ }^{5}$ Department of Computer Science, University of Copenhagen, Copenhagen, Denmark. ${ }^{6}$ Center for Lysosomal and Metabolic Diseases, Department of Paediatrics, Erasmus MC, University Medical Center Rotterdam, Rotterdam, The Netherlands.

Received: 5 July 2020 Accepted: 27 November 2020

Published online: 07 January 2021

\section{References}

1. van der Beek NA, de Vries JM, Hagemans ML, Hop WC, Kroos MA, Wokke $\mathrm{JH}$, et al. Clinical features and predictors for disease natural progression in adults with Pompe disease: a nationwide prospective observational study. Orphanet J Rare Dis. 2012;7:88.

2. van der Ploeg AT, Reuser AJ. Pompe's disease. Lancet. 2008;372(9646):1342-53.

3. van den Hout HM, Hop W, van Diggelen OP, Smeitink JA, Smit GP, Poll-The BT, et al. The natural course of infantile Pompe's disease: 20 original cases compared with 133 cases from the literature. Pediatrics. 2003;112(2):332-40.

4. Fromageot C, Lofaso F, Annane D, Falaize L, Lejaille M, Clair B, et al. Supine fall in lung volumes in the assessment of diaphragmatic weakness in neuromuscular disorders. Arch Phys Med Rehabil. 2001;82(1):123-8.

5. van der Beek NA, van Capelle Cl, van der Velden-van Etten Kl, Hop WC, van den Berg B, Reuser AJ, et al. Rate of progression and predictive factors for pulmonary outcome in children and adults with Pompe disease. Mol Genet Metab. 2011;104(1-2):129-36.

6. van der Ploeg AT, Clemens PR, Corzo D, Escolar DM, Florence J, Groeneveld GJ, et al. A randomized study of alglucosidase alfa in late-onset Pompe's disease. N Engl J Med. 2010;362(15):1396-406.

7. Stepien KM, Hendriksz CJ, Roberts M, Sharma R. Observational clinical study of 22 adult-onset Pompe disease patients undergoing enzyme replacement therapy over 5years. Mol Genet Metab. 2016;117(4):413-8.

8. Angelini C, Semplicini C, Ravaglia S, Bembi B, Servidei S, Pegoraro E, et al. Observational clinical study in juvenile-adult glycogenosis type 2 patients undergoing enzyme replacement therapy for up to 4 years. J Neurol. 2012;259(5):952-8.

9. Kuperus E, Kruijshaar ME, Wens SCA, de Vries JM, Favejee MM, van der Meijden JC, et al. Long-term benefit of enzyme replacement therapy in Pompe disease: a 5-year prospective study. Neurology. 2017.

10. Harlaar L, Hogrel JY, Perniconi B, Kruijshaar ME, Rizopoulos D, Taouagh $\mathrm{N}$, et al. Large variation in effects during 10 years of enzyme therapy in adults with Pompe disease. Neurology. 2019;93(19):e1756-67.

11. van der Ploeg AT, Kruijshaar ME, Toscano A, Laforet P, Angelini C, Lachmann $\mathrm{RH}$, et al. European consensus for starting and stopping enzyme replacement therapy in adult patients with Pompe disease: a 10-year experience. Eur J Neurol. 2017;24(6):768-e31.

12. Johnson EM, Roberts M, Mozaffar T, Young P, Quartel A, Berger KI. Pulmonary function tests (maximum inspiratory pressure, maximum expiratory pressure, vital capacity, forced vital capacity) predict ventilator use in late-onset Pompe disease. Neuromuscul Disord. 2016;26(2):136-45.

13. Berger KI, Chan Y, Rom WN, Oppenheimer BW, Goldring RM. Progression from respiratory dysfunction to failure in late-onset Pompe disease. Neuromuscul Disord. 2016;26(8):481-9.

14. Harlaar L, Ciet P, van der Ploeg AT, Brusse E, van der Beek N, Wielopolski PA, et al. Imaging of respiratory muscles in neuromuscular disease: a review. Neuromuscul Disord. 2018;28(3):246-56.

15. Wens SC, Ciet P, Perez-Rovira A, Logie K, Salamon E, Wielopolski P, et al. Lung MRI and impairment of diaphragmatic function in Pompe disease. BMC Pulm Med. 2015;15:54

16. Gaeta M, Barca E, Ruggeri P, Minutoli F, Rodolico C, Mazziotti S, et al. Late-onset Pompe disease (LOPD): correlations between respiratory muscles CT and MRI features and pulmonary function. Mol Genet Metab. 2013;110(3):290-6.

17. Gaeta M, Musumeci O, Mondello S, Ruggeri P, Montagnese F, Cucinotta $M$, et al. Clinical and pathophysiological clues of respiratory dysfunction 
in late-onset Pompe disease: new insights from a comparative study by MRI and respiratory function assessment. Neuromuscular Disord. 2015;25(11):852-8.

18. Mogalle K, Perez-Rovira A, Ciet P, Wens SC, van Doorn PA, Tiddens HA, et al. Quantification of diaphragm mechanics in pompe disease using dynamic 3D MRI. PLoS ONE. 2016;11(7):e0158912.

19. American Thoracic Society/European Respiratory Society. ATS/ERS Statement on respiratory muscle testing. Am J Respir Crit Care Med. 2002:166(4):518-624.

20. Quanjer PH, Stanojevic S, Cole TJ, Baur X, Hall GL, Culver BH, et al. Multiethnic reference values for spirometry for the 3-95-year age range: the global lung function 2012 equations. Eur Respir J. 2012;40(6):1324-43.

21. Wilson SH, Cooke NT, Edwards RH, Spiro SG. Predicted normal values for maximal respiratory pressures in caucasian adults and children. Thorax. 1984;39(7):535-8.

22. Yushkevich PA, Piven J, Hazlett HC, Smith RG, Ho S, Gee JC, et al. User-guided 3D active contour segmentation of anatomical structures: significantly improved efficiency and reliability. Neuroimage. 2006;31(3):1116-28

23. Koo TK, Li MY. A guideline of selecting and reporting intraclass correlation coefficients for reliability research. J Chiropr Med. 2016;15(2):155-63.

24. Barnard AM, Lott DJ, Batra A, Triplett WT, Forbes SC, Riehl SL, et al. Imaging respiratory muscle quality and function in Duchenne muscular dystrophy. J Neurol. 2019

25. Confalonieri M, Vitacca M, Scala R, Polverino M, Sabato E, Crescimanno $\mathrm{G}$, et al. Is early detection of late-onset Pompe disease a pneumologist's affair? A lesson from an Italian screening study. Orphanet J Rare Dis. 2019;14(1):62
26. Guimaraes MJ Winck JC Conde B, Mineiro A, Raposo M, Moita J, et al. Prevalence of late-onset pompe disease in Portuguese patients with diaphragmatic paralysis_DIPPER study. Rev Port Pneumol (2006). 2017;23(4):208-15.

27. Gayan-Ramirez G, Decramer M. The Respiratory Muscles. In: Grippi MA, Elias JA, Fishman JA, Kotloff RM, Pack Al, Senior RM, et al., editors. Fishman's pulmonary diseases and disorders, 5e. New York, NY: McGraw-Hil Education; 2015.

28. McCool FD, Tzelepis GE. Dysfunction of the diaphragm. N Engl J Med. 2012;366(10):932-42.

29. Mizuno M. Human respiratory muscles: fibre morphology and capillary supply. Eur Respir J. 1991;4(5):587-601.

30. Polla B, D'Antona G, Bottinelli R, Reggiani C. Respiratory muscle fibres: specialisation and plasticity. Thorax. 2004;59(9):808-17.

31. van den Berg LE, Drost MR, Schaart G, de Laat J, van Doorn PA, van der Ploeg AT, et al. Muscle fiber-type distribution, fiber-type-specific damage, and the Pompe disease phenotype. J Inherit Metab Dis. 2013;36(5):787-94.

32. Raben N, Roberts A, Plotz PH. Role of autophagy in the pathogenesis of Pompe disease. Acta Myol. 2007:26(1):45-8.

33. Ruggeri P, Lo Monaco L, Musumeci O, Tavilla G, Gaeta M, Caramori G, et al. Ultrasound assessment of diaphragm function in patients with late-onset Pompe disease. Neurol Sci. 2020;41(8):2175-84.

\section{Publisher's Note}

Springer Nature remains neutral with regard to jurisdictional claims in published maps and institutional affiliations.
Ready to submit your research? Choose BMC and benefit from:

- fast, convenient online submission

- thorough peer review by experienced researchers in your field

- rapid publication on acceptance

- support for research data, including large and complex data types

- gold Open Access which fosters wider collaboration and increased citations

- maximum visibility for your research: over $100 \mathrm{M}$ website views per year

At BMC, research is always in progress.

Learn more biomedcentral.com/submissions 